\title{
Neuer Fokus Viabilität
}

\author{
Menschliche Einschätzungen und Präferenzen sind veränderlich. Nevere philoso- \\ phische und neurowissenschaftliche Ansätze betonen, dass das Individuum auch \\ seine Außenwelt konstruiert, diese also nicht objektiv gegeben ist. Bezüge zur \\ Evolutorischen Ökonomik lassen sich leicht herstellen. Und entgegen entspre- \\ chender Kritik lässt sich auch auf dieser Ausgangsbasis eine Ethik denken, in der \\ Nachhaltigkeit als gesellschaftliche Viabilitätsbedingung gewährleistet wird.
}

\section{Von Marco Lehmann-Waffenschmidt} „Tom erschien auf dem Bürgersteig mit einem Eimer Tünche und einem langstieligen Pinsel. Er besah den Zaun, und aller Frohsinn verließ ihn... Bald mußten die anderen Jungs hier vorbeikommen, und sie würden ihn furchtbar auslachen, weil er arbeiten mußte... Er holte seine weltlichen Schätze aus der Tasche und betrachtete sie ..., es war nicht genug, um sich auch nur eine halbe Stunde echte Freiheit zu erhandeln, und so gab er den Gedanken an den Versuch wieder auf, einen der Jungs zur Mitarbeit zu kaufen. In diesem düsteren, hoffnungslosen Augenblick durchfuhr ihn eine Eingebung, nicht mehr und nicht weniger als eine grandiose, fabelhafte Eingebung!“

Es ist diese Eingebung Tom Sawyers in Mark Twains ,Die Abenteuer von Tom Sawyer und Huckleberry Finn“, die uns direkt zum Gegenstand dieses Beitrags führt. „Tom nahm seinen Pinsel und machte sich ruhig ans Werk. Bald kam Ben Rogers in Sicht - genau der, vor dessen Spott sich Tom am meisten gefürchtet hatte. ,Möchtest Du nicht lieber zum Schwimmen mitkommen? Aber Du möchtest natürlich lieber schuften, oder?' ,Was nennst Du denn Arbeit?' erwiderte Tom, ,Ich weiß nur eins, mir gefällt's.... Kriegt ein Junge vielleicht jeden Tag die Gelegenheit, einen Zaun zu streichen?' Das warf ein völlig neues Licht auf die Sache, ... und nach einer Weile sagte Ben: ,Tom, laß mich doch auch mal ein bißchen streichen.' ... Immer wieder schlenderten Jungen vorbei, sie kamen zum Spotten und blieben zum Tünchen ... Am Nachmittag bedeckte den Zaun eine dreifache Schicht Farbe, und Tom hatte die ganze Zeit über im Schatten gelegen und hübsch gefaulenzt." (1)

\section{- Die konstruktivistische Position}

Mark Twain spricht in seiner Geschichte „Der glorreiche Anstreicher" einen der Ecksteine der (radikal) konstruktivistischen Position an: Persönliche Einschätzungen und Präferenzen - in diesem Fall für die Art einer angemessenen Freizeitgestaltung - sind mentale Konstruktionen und können sich, auch wenn sie bisher invariant und stabil waren, plötzlich und radikal wandeln. Aber der radikal konstruktivistische Ansatz geht noch einen wesentlichen Schritt weiter. Nicht nur die eigenen Ansichten und Präferenzen, also die ,innere Welt" eines Subjekts, sind nach diesem Standpunkt von diesem Subjekt konstruiert, sondern auch alle seine Beobachtungen und Wahrnehmungen der ,äußeren Welt“. Unter Konstruktivismus wird in diesem Beitrag der konstruktivistische Ansatz in der Erkenntnistheorie und Neurowissenschaft verstanden. Als wichtigste Begründer werden Heinz von Förster, Ernst von Glasersfeld, Humberto Maturana und Francisco Varela angesehen. Als einfache alltagspraktische Beweise für die radikal konstruktivistische Position werden unter anderem optische Täuschungen (Vexierbilder), die Nichtwahrnehmung des Individuums des eigenen blinden Flecks im Sehfeld oder Phantomschmerzen angeführt.

Untermauerungen für diese Position finden sich auf zwei Ebenen: einmal auf der spekulativen Ebene in der philosophisch-erkenntnistheoretischen Tradition des Relativismus und zum anderen auf der empirisch-naturwissenschaftlichen Ebene in den neuen Erkenntnissen der Neuround Kognitionswissenschaften:

1. Die relativistische Position in der Erkenntnistheorie ist die logische Folge der Ablehnung der realistischen Position, die von einer objektiv existierenden und prinzipiell Beobachter-unabhängig erkennbaren absoluten Wirklichkeit ausgeht (2). Nach relativistischer Position gibt es keine „Realität“", die unabhängig vom Denken und Erkennen der Subjekte existieren könnte. Alle Aussagen über die Realität sind Schöpfungen, Konstruktionen von Subjekten. Demnach kann menschliches Erkennen nicht einfach, wie zum Beispiel von der in den Sozialwissenschaften nach wie vor populären Position des Kritischen Rationalismus postuliert, auf eine ständig verbesserte Wahrheitsapproximation zielen, da das vermeintliche Objekt dieser versuchten Wahrheitsapproximation so nicht existiert. Beispielsweise kann sich das prognostizierte Verhalten einer Gruppe allein schon dadurch verändern und die Prognose als „self-fulfilling prophecy“ überhaupt erst zutreffen lassen, dass die Prognose den involvierten Entscheidungs- und Handlungsträgern im voraus bekannt wird (3). Auch im Rahmen der Makroökonomie können die Ansichten der Akteure über das Funktionieren der Wirtschaft eine bestimmende Rolle für ihr Funktionieren spielen (4). In Anlehnung an die Heisenbergsche Unschärferelation könnte man von einer ,Sozialwissenschaftlichen Unschärferelation" sprechen, indem in beiden Fällen der Beobachter allein schon durch seine Beobachtung den Beobachtungsgegenstand verändert oder ihn sogar erst konstituiert.

2. Auf einen kurzen Nenner gebracht lauten die naturwissenschaftlichen Argumente für die konstruktivistische Position, dass alle neuro(physio)logischen Prozesse der wahrnehmungssensorischen Peripheriefunktionen beim Menschen wegen ihrer physikalisch-chemischen Eigenschaften keine spezifischen Bedeutungsinhalte übertragen können (5). Sie sind lediglich unspezifische elektrochemische Signale, denen erst im Gehirn inhaltliche Bedeutungen zugemessen werden. Das heißt, sie sind keine Repräsentationen einer absoluten Wirklichkeit, sondern werden erst in einem abgeschlossenen System, dem menschlichen Gehirn, auf selbstorganisierte Weise (autopoietisch) zu für das Subjekt bedeutungstragenden Konstruktionen.

\section{Homo oeconomicus obsolet}

Neueste neurobiologische Ergebnisse gehen allerdings noch einen Schritt weiter (6). Demnach ergeben sich auf die Frage nach der Willens- und Entscheidungsfreiheit menschlichen Handelns weit weniger Freiheitsgrade als bisher angenommen. Statt - zumindest im Idealfall bei ausreichender Zeit und unter den richtigen Anreizmechanismen - reflektierende und Alternativen kalkulierende Subjekte zu sein, scheinen menschliche Akteure bei ihren „Wahlhandlungen“ grundsätzlich von habituellen Verhaltensmustern gesteuert zu werden, die zum großen Teil in phylogenetisch alten Gehirnstrukturen abgelegt sind. Gleichzeitig suggeriert das Gehirn aber dem Akteur, er handele auf Grund einer von ihm kalkulierten freien Willensentscheidung, sei also Sub- 
jekt und nicht nur Ausführender. Damit erscheint nicht nur die Vorstellung des Homo oeconomicus, sondern jede Vorstellung eines Alternativen gegeneinander abwägenden und daraus sein Verhalten ableitenden Subjekts in der bisherigen Form als obsolet. Allerdings widerspricht dies nicht einer grundsätzlichen längerfristigen Wandlungsfähigkeit der verhaltenssteuernden habituellen Verhaltensmuster etwa durch Lernprozesse. Auf einer längerfristigen Zeitskala könnte so die menschliche Kalkulationsfähigkeit als zumindest partiell zurïckgewonnen betrachtet werden.

Ein wichtiges Element der konstruktivistischen Position, das sie auch von den meisten anderen relativistischen Ansätzen unterscheidet, ist ihre pragmatische Ausrichtung. Der radikal konstruktivistische Ansatz begnügt sich nicht damit, einen allgemeinen Wahrnehmungs- und Bedeutungsrelativismus zu konstatieren und die Wahrheitsbzw. Wirklichkeitsapproximationsfähigkeit menschlichen Erkennens in Frage zu stellen. Er stellt seine Aussagen vielmehr mit dem so genannten Viabilitätskonzept in einen konkreten menschlichen Lebensbezug und lässt sie damit sowohl für die Wirtschaftswissenschaft, und dort insbesondere für den evolutorischen Ansatz, als auch für die Nachhaltigkeitsdebatte relevant werden.

\section{- Nicht optimal, sondern viabel}

Kurz gesagt bedeutet das Viabilitätskonzept ein differenziertes Selektionskonzept, wonach alle menschlichen kognitiven Konstruktionsleistungen keineswegs eine zweckfreie Existenz haben, sondern zwangsläufig einer ständigen Bewertung unterliegen, indem sie sich bewähren und zu viablen, also das Überleben garantierenden, Ergebnissen für das konstruierende Subjekt führen müssen. Die konstruktivistische Position steht durch das Viabilitätskonzept offensichtlich dem ökonomischen Denken nahe und hier insbesondere dem evolutorischen Ansatz (7). Denn nicht - eventuell schwer erreichbare und nur unzulänglich verifizierbare - Optimalitätskriterien an die kognitiven Konstruktionen eines Akteurs stellen Maßstab und Richtschnur für dessen weitere Entwicklung dar, sondern die Fähigkeit, mit seinen Konstruktionen in seiner Lebenswelt ,durchzukommen", also viabel zu sein.

Das Viabilitätskriterium liefert auch das wichtigste Argument gegen die Kritik, der konstruktivistische Ansatz widerlege sich in der reflexiven Anwendung selbst: Wenn es keine Subjekt-unabhängigen wahren Aussagen gibt, dann könne man auch di- ese Kernaussage der konstruktivistischen Position sowie alle „Beweise“ - auch die naturwissenschaftlich-empirischen - nicht als objektiv wahr bezeichnen. Diese Kritik übersieht aber das eigentliche Anliegen des konstruktivistischen Ansatzes. Denn das Gütekriterium jeder Konstruktion, also auch des Konstruktivismus, ist die Eigenschaft der Viabilität, nicht die der Wahrheit oder einer Wahrheitsapproximation.

\section{- Die Universaliendebatte}

Das Viabilitätskonzept kann man auch als Antwort auf den so genannten Solipsismus-Vorwurf gegen den konstruktivistischen Ansatz ansehen. Demnach impliziert der konstruktivistische Ansatz durch das Postulat der kognitiven Abgeschlossenheit jedes Individuums und der von ihm solitär konstruierten Welt eine völlige Individualisierung und Beliebigkeit (Partikularisierung) menschlicher Wahrnehmung und Werthaltungen, was letztlich das Auseinanderfallen menschlicher Gemeinschaften zur Folge haben müsste. Dies sei aber so nicht zu beobachten, und damit sei der konstruktivistische Ansatz widerlegt. Bei dieser Argumentation wird allerdings übersehen, dass die konstruktivistische Position keineswegs denknotwendig eine völlige Individualisierung und Beliebigkeit menschlicher Wahrnehmungen und Werthaltungen impliziert. Vielmehr besagt ja gerade das Viabilitätskonzept, dass Konstruktionen immer einer Bewährungsprobe ausgesetzt sind und mit über die weitere Existenz des betreffenden Subjekts entschieden wird. Zudem nimmt der konstruktivistische Ansatz das Zustandekommen von Konventionen und anderen gesellschaftlichen Verhaltensmustern als genuinen Bestandteil menschlicher Gemeinschaften in sein Erklärungskonzept mit auf. So zielt der so genannte Universalienansatz als Teil des konstruktivistischen Ansatzes auf die Erklärung, wie soziale Prozesse in allen menschlichen Lebensbereichen Universalien in Form von gemeinsamen Wert- und Verhaltensmustern erzeugen können (8).

\section{Zur Ethik des Konstruktivismus}

An den Solipsismus-Vorwurf schließt sich unmittelbar die Frage nach dem Verhältnis des konstruktivistischen Ansatzes zur Ethik an. Auch hier kann dem scheinbar nahe liegenden Argument, die konstruktivistische Position bestärke eine Fragmentierung der Gesellschaft durch Individualisierung und trage damit zum moralischen Relativismus und zur Auflösung ethischer Prinzipien bei, schon durch den Hinweis auf den Universalienansatz begegnet werden. Der konstruktivistische Ansatz bietet aber über dieses Argument hinaus noch eine weitere Bestärkung der ethischen Position. Die Grundlage dafür bildet die Einsicht, dass ein Subjekt, das seine kognitiven Inhalte selbst konstruiert, in maximaler Weise eigenverantwortlich ist für ein an ethischen Prinzipen orientiertes Verhalten (9). Konkretisiert wird dieses Prinzip Freiheit bedeutet Verantwortung in der konstruktivistischen Ausprägung des Kantschen Imperativs, dem von Heinz von Foerster postulierten „Konstruktivistischen Imperativ“. Er misst die gesellschaftliche Bedeutung individuellen Handelns daran, ob durch dieses Handeln die Handlungsoptionen anderer Akteure vermehrt werden. Interessant ist, daß der Konstruktivistische Imperativ inhaltlich übereinstimmt mit dem „Evolutorisch-ökonomischen Imperativ“, der ökonomisches Handeln, insbesondere des Staates und von Institutionen, danach beurteilt, ob dadurch die Variabilität der Handlungsmöglichkeiten der ökonomischen Akteure gesteigert wird.

\section{Relevanz für die Nachhaltigkeitsdebatte}

Die Schlussfolgerungen aus diesen Überlegungen für die Nachhaltigkeitsdebatte sind offensichtlich. Man kann das Erfordernis nachhaltigen Verhaltens geradezu als paradigmatischen Anwendungsfall des Konstruktivistischen Imperativs sehen, und zwar sowohl auf der Ebene des Individuums als auch auf der gesellschaftlichen Ebene. Darüber hinaus besteht aber auch eine weitere direkte Beziehung des konstruktivistischen Ansatzes zur Nachhaltigkeitsproblematik auf der Ebene der Gesellschaft. Auch hier spielt wieder das Viabilitätskonzept eine entscheidende Rolle. Sind doch alle im Zusammenhang mit dem Nachhaltigkeitsproblem relevanten Messungen, Beobachtungen, Wahrnehmungen und Bewertungen zwangsläufig menschliche Konstruktionen und unterliegen damit dem Viabilitätskriterium. Ein Beispiel ist die öffentliche Diskussion über eine mögliche anthropogene Verursachung des Klimawandels. Nur nachhaltiges Verhalten kann per definitionem viabel sein - wenn nicht im kleinen Zeitfenster des Individuums, so doch im großen Zeitfenster der Gesellschaft, oder der Menschheit, als Ganzer. Dass das Viabilitätskriterium damit in der Nachhaltigkeitsproblematik in erster Linie auf der Gruppen- bzw. Gesellschaftsebene wirksam wird, bringt jedoch zusätzliche Komplexität mit sich. Denn das nachhaltige Wohlergehen eines Individuums hängt nun nicht nur von seinem eigenen Verhalten, sondern vor allem auch vom Verhalten 
aller anderen Akteure ab. Als interdependent-interaktives Problem fällt die Nachhaltigkeitsproblematik damit auch in die Domäne der Spieltheorie und ihren neuen empirisch-experimentellen Zweig. Hier schließt sich der Kreis zum Beitrag von Gächter in diesem Themenschwerpunkt, in dem mit der Untersuchung von Aspekten des Kooperationsverhaltens wie Trittbrettfahren, Fairness und Reziprozität die spieltheoretische Feinstruktur der Nachhaltigkeitsproblematik behandelt wird.

\section{Anmerkungen}

(1) Twain, Mark: Die Abenteuer des Tom Sawyer und Huckleberry Finn, Zürich 2001 (Erstauflage 1876), S. 29-36.

(2) Vgl. z.B. Wendel, Hans-Jürgen: Moderner Relativismus. Zur Kritik antirealistischer Sichtweisen des Erkenntnisproblems, Tübingen 1990; Dettmann, Ulf: Der Radikale Konstruktivismus. Anspruch und Wirklichkeit einer Theorie, Tübingen 1999.

(3) Vgl. z.B. Lehmann-Waffenschmidt, Marco: Predictability of Economic Processes and the Morgenstern Paradox,

Schweizerische Zeitschrift für Volkswirtschaft und Statistik, 1990.

(4) Vgl. z.B. Henderson, David: Innocence and Design. The Influence of Economic Ideas on Policy, Oxford 1986; Meier, Alfred/Slembeck, Tilman: Wirtschaftspolitik. Kognitiv-evolutionärer Ansatz, München/Wien 1998, 2. Aufl.

(5) Vgl. z.B. Rusch, Gebhard/Schmidt, Siegfried/ Breidbach, Olaf: Interne Repräsentationen, Frankfurt 1996.

(6) Pauen, Michael und Gerhard Roth (Hrsg.): Neurowissenschaften und Philosophie, München 2001.

(7) Vgl. Ötsch, Walter: Die Herausforderung des Konstruktivismus für die ökonomische Theorie. In: Priddat, Birger P./ Wegner, Gerhard (Hrsg.): Zwischen Evolution und Institution. Neue Ansätze in der ökonomischen Theorie, Marburg 1996, S. 35-56; Lehmann-Waffenschmidt, Marco: Konstruktivismus und Evolutorische Ökonomik. In: Rusch, Gebhard (Hrsg.): Wirtschaftswissenschaften und Konstruktivismus, Frankfurt 2002.

(8) Hejl, Peter M.: Einleitung. Das Ende der Eindeutigkeit. In: Stahl, Heinz K./ Hejl Peter M. (Hrsg.): Management und Wirklichkeit. Das Konstruieren von Unternehmen, Märkten und Zukünften, Heidelberg 2000.

(9) Vgl. z.B. Rusch, Gebhard/ Schmidt, Siegfried: Konstruktivismus und Ethik, Frankfurt 1995.

\section{Der Autor}

Dr. Marco Lehmann-Waffenschmidt ist Professor für Volkswirtschaftslehre, insbes. Managerial Economics, an der Technischen Universität Dresden.

Kontakt: TU Dresden, Fakultät Wirtschaftswissenschaften, Mommsenstraße 13, 01062 Dresden.

Tel. 0351/ 463-32871, Fax -37285,

E-Mail: Iw@rcs.urz.tu-dresden.de
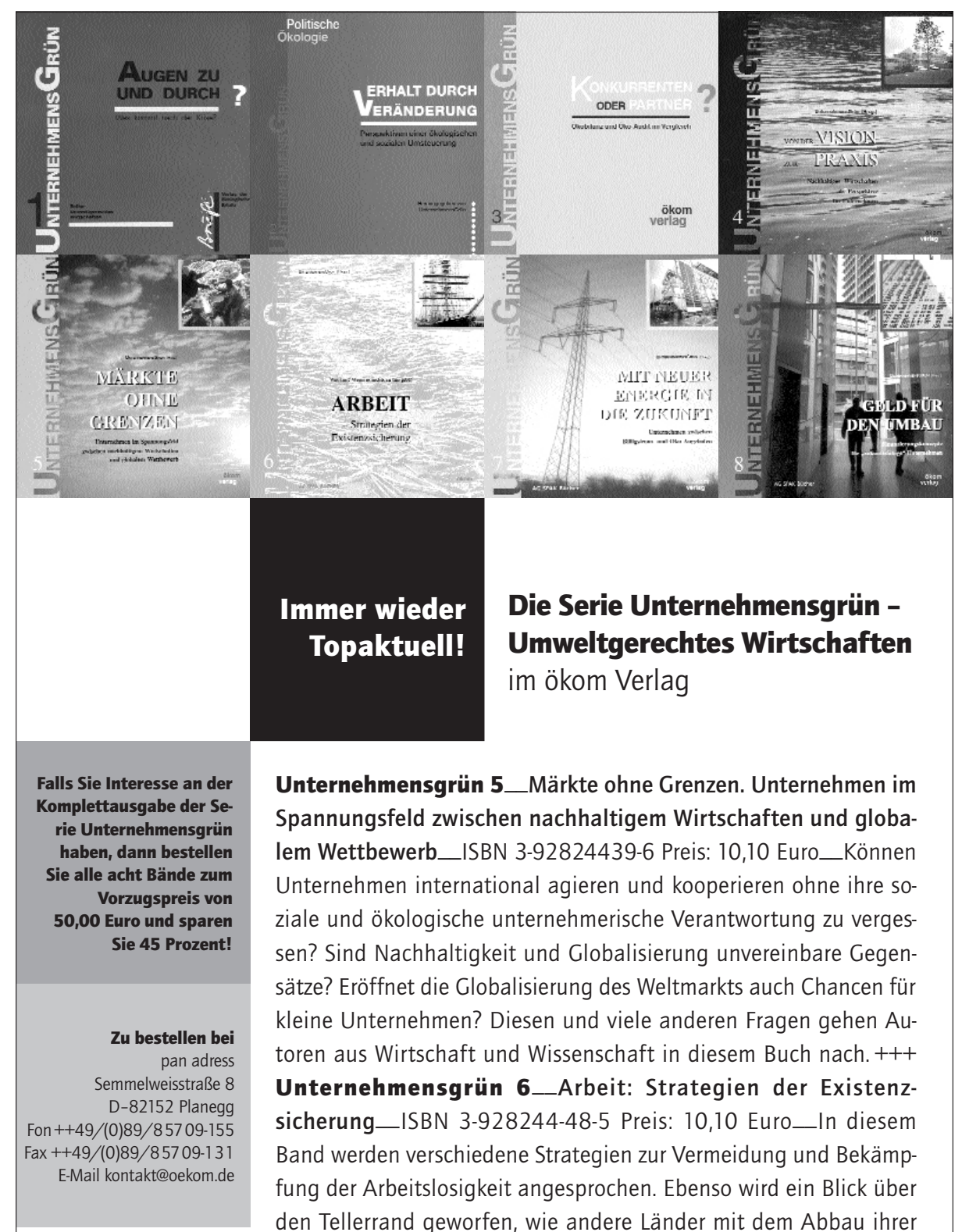

Unternehmensgrün 5_Märkte ohne Grenzen. Unternehmen im Spannungsfeld zwischen nachhaltigem Wirtschaften und globalem Wettbewerb_ISBN 3-92824439-6 Preis: 10,10 Euro_Können Unternehmen international agieren und kooperieren ohne ihre soziale und ökologische unternehmerische Verantwortung zu vergessen? Sind Nachhaltigkeit und Globalisierung unvereinbare Gegensätze? Eröffnet die Globalisierung des Weltmarkts auch Chancen für kleine Unternehmen? Diesen und viele anderen Fragen gehen Autoren aus Wirtschaft und Wissenschaft in diesem Buch nach. +++ Unternehmensgrün 6_Arbeit: Strategien der Existenzsicherung_ISBN 3-928244-48-5 Preis: 10,10 Euro_In diesem Band werden verschiedene Strategien zur Vermeidung und Bekämpfung der Arbeitslosigkeit angesprochen. Ebenso wird ein Blick über den Tellerrand geworfen, wie andere Länder mit dem Abbau ihrer Arbeitslosigkeit umgehen. Mit diesen Themen beschäftigen sich Autoren aus Politik und Wirtschaft. +++ Unternehmensgrün 1 Augen zu und durch? Was kommt nach der Krise? ISBN 3-92850705-2, Preis: 10,10 Euro +++ Unternehmensgrün 2_Erhalt durch Veränderung. Perspektiven einer ökologischen und sozialen Umsteuerung. ISBN 3-928244-13-2, Preis: 10,10 Euro +++ Unternehmensgrün 3_Konkurrenten oder Partner. Ökobilanz und ÖkoAudit im Vergleich. ISBN 3-928244-16-7, Preis: 10,10 Euro +++ Unternehmensgrün 4_Von der Vision zur Praxis. Nachhaltiges Wirtschaften als Perspektive für Unternehmen. ISBN 3-92824427-2, Preis: 15,30 Euro +++ Unternehmensgrün 7_Mit neuer Energie in die Zukunft. ISBN 3-928244-66-3, Preis: 12,80 Euro+++ Unternehmensgrün 8_Geld für den Umbau. ISBN 3-92824473-6, Preis: 12,80 Euro. 
(c) 20I0 Authors; licensee IÖW and oekom verlag. This is an article distributed under the terms of the Creative Commons Attribution Non-Commercial No Derivates License (http://creativecommons.org/licenses/by-nc-nd/3.o/), which permits unrestricted use, distribution, and reproduction in any medium, provided the original work is properly cited. 